\title{
Communication Technologies in a Remote Workplace
}

\author{
Sarma CAKULA, Madara PRATT \\ Vidzeme University of Applied Sciences \\ Cēsu iela 4, Valmiera, LV - 4201, Latvija \\ sarma.cakula@va.lv, madara.pratteva.lv
}

\begin{abstract}
The global pandemic, which began in 2020, ushered in a significant time in the field of information technology, when the performance of work duties, the receipt of services and mutual communication became most directly dependent on information and communication technologies. This new digital work environment poses new problems and challenges for business leaders. In addition, it must be taken into account that employees may experience stress as a result of the changes caused by the pandemic and the active use of information and communication technologies, which may result in psychological difficulties as a side factor, must be taken into account. The main goal of this study is to create a effective communication in digital workplace model that includes factors for effective communication in forced distancing conditions, as well as to provide recommendations for companies. The model consists of objectives, capabilities and contextual elements for an efficient communication system in forced distancing conditions. The model has been developed using the 4EM method, describing the factors involved and the attributes to be measured. On the basis of the model, a a communication system had been created, which will promote an efficient, safe and motivating work environment online and helps for company leaders (i.e. teleworking). As a large part of communication, efficiency depends not only on the used communication system (i.e. information and communication technologies) but also on the competence of managers. Recommendations for company managers to improve communication efficiency are developed and provided.
\end{abstract}

Keywords: Technology-based communication, Work motivation, System modelling, Automated estimation.

\section{Introduction}

Most of the human interaction is non-verbal and the communication using technologybased means is not as effective and face-to-face meetings. It must be remembered that in communication which is based on information and communication technologies, not only the technological aspects are essential, but also the ability of management and employees to communicate effectively in this way. Not all people have a natural talent for using different forms of communication and doing so effectively. People's ability to adapt is especially critical when communicating online. When using information and communication technologies, communication is diverse. In addition, not all communication is verbal. It should also be borne in mind that communication also takes 
place non-verbally and that these additional non-verbal signals can't always be transmitted or are transmitted using information and communication technologies. Helander (2014) pointed out that human-computer interaction research should strive to understand what are the effects of the use of computers on people's productivity, job satisfaction, communication with other people, and the general quality of their lives (Helander, 2014). There is a new set of complex challenges in organizations when face-to-face communication is transferred to technology-based communication (Pratt et al., 2020c).

Not all people have the natural ability for effective communication. This is especially critical within online communication. Tusubira and Mulira (2004) name lack of awareness, mindset and lack of top-level commitment as main sources of implementation problems and suggest to develop a systematic method of ICT implementation (Tusubira, 2004).

In the last decade information and communication technologies have an immense impact on the opportunities and constraints for social development. They play an increasingly important role in the daily life of society, in mutual communication and the operation of companies. In addition, technologies are constantly evolving and offer ever wider and more comprehensive capabilities.

We have been using intelligent assistants and chatbots for some time. The main task of such smart helpers is to be able to comprehend what a person is saying or writing to him and to give an appropriate answer or take action according to a programmed logic. And so more and more companies are using smart helpers to replace employees to communicate with customers. However, take into account the fact that people will not always choose to communicate with smart helpers. They will want to communicate with people, even if they have to wait, it will cost more. As a result, more and more companies are working to develop individual smart assistants (so-called digital twins) that can open up unprecedented business and collaboration opportunities. In addition, digital twins can perform other business-friendly activities. For example, collect real-time data, identify key events affecting business, at the right time, objectively pass information to the recipient. Thus, digital twins can also be a valuable decision support tool, indicated by the research team (Yan Hong Lim, 2020). Accordingly, the paper uses the definition of the term 'digital twin' given by Sandkuhl and Stirna (Sandkuhl et al., 2018): "a dynamic virtual representation of a physical object or system across its lifecycle, using real-time data to enable understanding, learning and reasoning".

The main challenges and problems to effective communication relate to the availability of technology (mainly devices, software and internet), the functionality of selected communication tools, employee skills, the ability of company management to respond to change and the ability to communicate remotely. As a result, the use of technology in communication has several consequences (positive and negative ones). In order to reduce the occurrence of such problems, the company must have an action plan on how to work in a remote work situation. Thus, the company would have taken preventive measures to protect itself and its employees from incidents.

The goal of the paper is to create a model for effective communication in the conditions of forced distance. 


\section{Theoretical Background}

\subsection{Digital Communication}

Coming of new technologies has many advantages to organizations. Communication can be organized in four different settings according to time and place (Mittleman et al.,1999; Levi, 2014). Digital communication happens without the form of face-to-face meetings. It could be managed by video, audio or text. There are synchronized meetings online for all in the same time or out of sync in different time when a meeting is recorded in a storage place and can be used for employees with different time shifts. In this type of meeting participants share the same virtual space and web. Each of these settings can be applied depending on the situation, purpose, availability, resources, and context of transmitted message. It is critical for management to decide which of media is most appropriate to use in a every situation. Within Media Richness Theory, different means of technology are arranged from leaner medium channels to richer medium channels. Phone calls and video calls are considered as effective channels, but the most effective way of communications are face-to-face meetings. Face-to-face meetings provide high bandwidth for the communication. According to this theory, managers have to be very careful about the choices of communication tool they use in each situation. Leaner medium channels can be used for general announcements, but severe and problem-solving issues require richer medium channels (Workman, 2003).

\subsection{Research results}

In Pauga and Cakula (2019) research e-mails and phone calls are the most popular means of work communication, even though various new technologies are available (Pauga et al.., 2019). However, in another study, video-conferencing was not found to be an equal substitute for face-to-face meetings (Thompson et al., 2006). Video-conferencing brings the image together with the name of participant, but still misses necessary non-verbal cues for effective communication. A considerable amount of communication is non-verbal: kinesics (i.e., facial expressions, gestures, body movement); proxemics (i.e., perception and use of space); chronemics (i.e., perception and use of time) (Burgoon et al., 2011). Missing facial expressions and non-verbal cues cause lack of interpersonal relations, social rapport and lead to less satisfied members (Levi, 2017). It becomes harder to know if the information was under-stood fully or correctly, and can be challenging to establish and maintain mutual knowledge (Driskell et al., 2003). These problems are more complex across different cultures and can lead to false conclusions and assumptions about a person's character (Levi, 2017; Vignovic, 2010).

Two sets of empirical data were used in this study. Pratt and Cakula (2021) used data set from 2019 for motivation assessment survey with 102 respondents from 26 different countries (Pratt et al., 2021a). Second, open data from an open database with 35 different measurable attributes and 1470 surveyed employees in the IT company were used to measure parameters such as employee satisfaction, productivity and turnover (Pratt et al., 2021b).

Technology-based communication system developers have to consider many constructs. The model will be a guide for the development of more effective communication systems and include some of the necessary constructs (Pratt et al., 2020b). 
One of the main goals of effective communication is motivated and satisfied employees. Employee motivation and satisfaction are essential aspects of remote work, especially at a time when the use of information and communication technologies has not been a key objective so far. At the same time, it must be remembered and taken into account that the psychological well-being of employees is an integral part of the quality of work.

Remote working must be organized in such a way as to ensure that it is not only efficient, does not create additional stress and strain on employees, but is also safe.

\section{Description of the model}

The model is based on research in various fields and the experience and knowledge of the authors. The main task of this model is to describe and include the necessary factors for effective communication within a remote working situation.

Accordingly, these are factors that should be provided by an efficient communication system and tool.

The model includes four important objectives:

- communicate effectively;

- motivated and satisfied employees in exceptional circumstances.

- The model lists the different capabilities of the communication tool:

- effective internal communication of employees;

- communication flexibility;

The service provided is a "Communication system". Every ability is based on different contexts.

The goal of the ability "Effective internal communication of employees" is to "Communicate effectively". This goal is ensured by the system which is a customized communication technology.

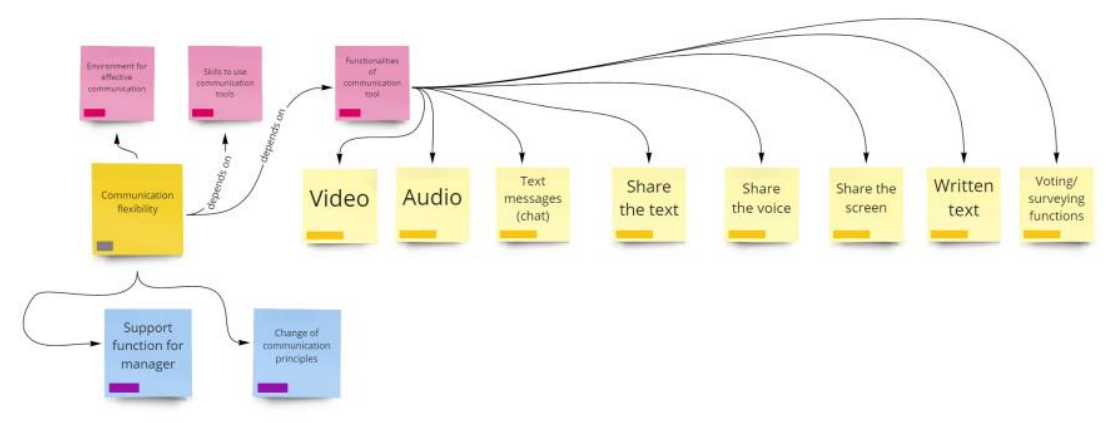

Fig. 1. The model of ability "communication flexibility"

This goal is supported by the ability to "Communication flexibility". It depends on three context elements (Fig. 1): 
functionalities of communication tool- these functions may include measurable attributes such as video, audio, text messages (chat), scree, text and voice sharing, written text, and voting/ surveying functions;

- $\quad$ skills to use communication tools;

- environment for effective communication.

There are two adjustments to "communication flexibility": change in communication principles and a support function for managers.

The goal of "Communicate effectively" can be measured by the following KPIs: "channel richness", average time between communications, average amount of communication over a period of time, message length depending on the context, and time spent on communication (Fig. 2). Accordingly, these KPIs derive the measurable attributes used for the digital twin: length of the communication time, number of communication events, average message length depending on the context, and time spent on communication.

Another goal of effective communication is motivated and satisfied employees in exceptional circumstances. It is measured by the level of employee satisfaction and motivation in the organization as a whole and these are measurable attributes. Another way to measure motivation and satisfaction is by using net promoters score.

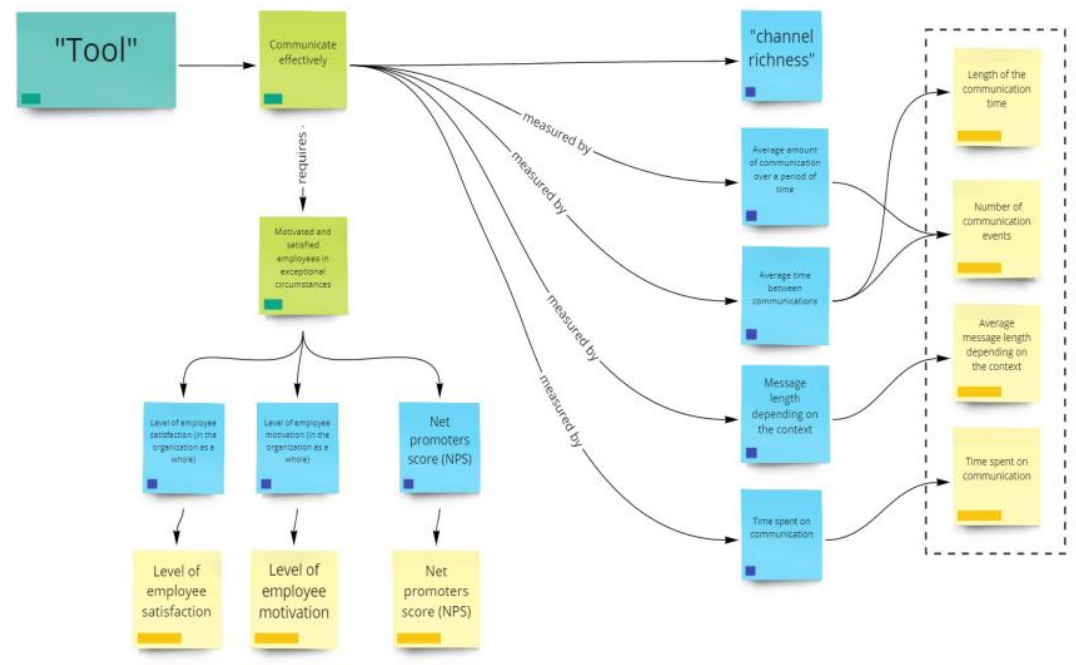

Fig. 2. The goal "Communicate effectively" model

The ability to "Effective and secure internal communication of employees" has three contextual elements:

1. Internet, which is measured by the number of interruptions per unit of time and the data transfer rate.

2. Electricity availability, measured by the number of interruptions per unit of time and the duration of the interruption. There are three possible adjustments to electricity availability:

if the break duration is $>\mathrm{X}+100$ then install the generator; 
if the number of breaks $>\mathrm{X}$ then install the universal power supply (UPS);

if "too often" then train employees in UPS and generator usage.

3. Percentage of employees working remote. The attributes to be measured are the number of employees and the number of employees working remote.

\section{Application of the model}

\subsection{Employee motivation level}

The system based on this model helps to solve several problems. One of the problem is when dissatisfied and unmotivated employees lead to lower productivity and can lead to employees leaving the company. Motivating employees in teleworking can be very difficult. As a solution, a system is offered that ensures the measurement of employees' mood every day by connecting to the communication system and regular surveys with the processing of results using an algorithm to take the necessary preventive measures. Measurable attributes:

- evaluation of management communication from the results of the employee survey;

- motivation;

- level of satisfaction and employee turnover.

Model input parameters:

- survey data results;

- employee turnover in the organization;

- employee performance appraisal.

Input parameter values:

$\bullet \%$ of employee turnover;

- employee evaluation results.

\subsection{Climate of effective communication in the organization}

The second problem in the context of teleworking, it may be less obvious whether communication is frequent, effective and appropriate to the situation.

Measurable attributes:

- duration of communication;

- number of communication events;

- average message length depending on the context;

- time spent on communication;

- evaluation of communication (i.e. evaluation of the usefulness of communication involved in communication, usefulness of tools used, etc.).

Input parameters:

- availability of communication tools;

- organizational conditions for communication.

Input parameter values:

- summary of available communication tools;

- parameters for quality communication provision; Guidelines: Defining guidelines for effective communication, implement company norms on the recommended frequency of the meeting / message exchange, the length of the message according to the context, the recommended duration of the meetings. 


\subsection{Leader support function}

The system gives also support for manager functions. The problem is that without the necessary communication functions, the communication efficiency may be reduced. Keywords: communication tool, functionality, technology-based communication. Main objective is to determine whether the organization has the various communication functions required for a teleworking situation to ensure that employees have access to all necessary communication tools and that they are used effectively, thus facilitating the transfer and perception of information to managers.

Measurable attributes:

- written text;

- video;

- Shared screen

- audio;

- Voice sharing

- Text sharing

- voting / polling functions;

- Text messages (chat).

System verifies that the technology support provided has the appropriate features.

Input parameter:

- availability of communication tools;

- organizational conditions for communication;

- managerial satisfaction with communication. Input parameter values:

- summary of available communication tools;

- parameters for quality communication provision;

- managers' assessment of the effectiveness of the tools used.

Digital twin and machine learning had been used for experimental results and validation (Lektauers et al., 2021). The Random Forest algorithm shows the best capabilities with accuracy 85.12. It is considered as good accuracy (Pratt et al., 2020a).

\section{Recommendations}

Recommendations on how to provide durable services in emergency situations:

- The company should develop guidelines for action in the event of forced distancing:

○ provide or discuss internet access and digital devices;

o provide employees with the necessary software;

- provide employees with secure access to systems and resources online;

○ train employees on minimum safety requirements when working online;

- train managers and employees to work online.

- To ensure effective communication:

- choose the communication channel according to the context;

- have regular communication with employees individually;

o organize regular online meetings;

$\circ$ create message lengths depending on the context;

- determine the time required for communication; 
- provide efficient channel access with file, voice, video and text messaging capabilities;

- provide managers with a support function.

- Guidelines and recommendations for communication in online company meetings and technology-based communication:

$\circ$ to encourage employees to communicate with each other informally on a daily basis;

○ use some of the social networks to build emotional capital;

○ ensure regular meetings;

○ well-defined meeting structure;

○ to acquaint with e-mail norms - structure, response time frames and addressees;

- draw attention to the impact of emotional state on electronic correspondence and other forms of communication (for example, emails should not be sent in a sad or emotionally agitated state);

- start the video conference with an introduction by the leader of the meeting and a review of the participants in the meeting (i.e. identification of who has/has not attended the meeting);

- during video conferencing no side conversations;

o ask the participants of the meeting to announce if there are any uncertainties.

Monitor the level of motivation and satisfaction of the company's employees through surveys and regular communication with employees.

Development of technologies and new ways of communication has given as many challenges as there are benefits. It connects people all over the world, gives more flexibility and increases speed of work, while the messages people convey can be losing meaning and context. Motivation is an important factor affecting employee satisfaction. However, motivating employees using technology-based communication is a challenge.

It has been discovered by Pratt and Cakula that leaders have main role in motivating employees and it can be done by both - motivating employees through face-to-face communication and through technology-based communication (Pratt, 2020a). Technological solutions for companies have to be offered and developers need guidance for new system development, which include social science research. The system based on developed model supports leaders in communication with their employees and allows to identify the overall volatility of the company.

\section{Conclusion}

Development of technologies and new ways of communication has given as many challenges as there are benefits. It connects people all over the world, gives more flexibility and increases speed of work, while the messages people convey can be losing meaning and context. Motivation is an important factor affecting employee satisfaction. However, motivating employees using technology-based communication is a challenge.

It has been discovered by Pratt and Cakula that leaders have main role in motivating employees and it can be done by both - motivating employees through face-to-face communication and through technology-based communication (Pratt, 2020a). Technological solutions for companies have to be offered and developers need guidance 
for new system development, which include social science research. The system based on developed model supports leaders in communication with their employees and allows to identify the overall volatility of the company.

\section{Acknowledgment}

Support for effective participation of Vidzeme University of Applied Sciences within the international scientific circles (ViA-lnt), project number 1.1.1.5/18/I/005

\section{References}

Burgoon, V., Guerrero, J. K, Manusov, L. K. (2011). Nonverbal signals, In: Terpers. Commun., SAGE Handb. 26,. 239-280.

Driskell, J. E., Radtke, P. H., Salas, E. (2014). Virtual Teams: Effects of Technological Mediation on Team Performance., In: Gr. Dyn. Theory, 7(4), 297-323.

Helander, M. G. (2014). Handbook of human-computer interaction. Elsevier.

Lektauers, A., Pecerska, J., Bolsakovs, V., Romanovs, A., Grabis, J., Teilans, A. (2021). A multimodel approach for simulation-based digital twin in resilient services. WSEAS Transactions on Systems and Control 16, pp.133- 145.

Levi D. (2014). Group dynamics for teams. SAGE.

Levi, D. (2017). Group dynamics for teams, 5th ed. Sage Publications.

Mittleman, D., Briggs, R. (1999). Supporting work team effectiveness : best management practices for fostering high performance | Search Results | IUCAT Kokomo, 1st ed.: Jossey-Bass Publishers, San Francisco.

Pauga, M., Cakula, S. (2019). Motivation in a business company using technology- based communication', In: International Journal of Computer Science Series, London, 1(1), 1-12.

Pratt, M., Boudhane, M., Cakula, S. (2021a). Predictive Data Analysis Model for Employee Satisfaction Using ML Algorithms. In: Saeed F., Al-Hadhrami T., Mohammed F., Mohammed E. (eds) Advances on Smart and Soft Computing. Advances in Intelligent Systems and Computing, Springer, Singapore, 1188.

Pratt, M., Cakula, S. (2020a). The Impact of Information and Communication Technologies (ITC) on Employees' Motivation, In: INTED2021 - International Education Conference Proceedings, INTED2020 Proceedings, pp. 1581-1589.

Pratt, M., Cakula, S., Majore, G., Buss E. (2020b). Development of an Effective and Secure Communication System in a Quarantine Situation. In: 2020 International Conference on Computational Science and Computational Intelligence (CSCI 2020). IEEE CPS. In press.

Pratt, M., Cakula, S. (2021b). Motivation in a business company using technology-based communication. In: Studies in Computational Intelligence, 928, 15-30.

Pratt, M., Cakula, S. (2020c). The Impact of Using Technology-Based Communication on Quality of Work Relationships. In: Baltic Journal of Modern Computing, University of Latvia, Riga, 8(1), 143-153.

Sandkuhl, K., Stirna J. (2018). Capability Management in Digital Enterprises. Springer International Publishing AG.

Thompson, L. F., Coovert, M. D. (2006). Understanding and Developing Virtual ComputerSupported Cooperative Work Teams. In: Creating high-tech teams: Practical guidance on work performance and technology, American Psychological Association, Washington, pp. 213-241.

Tusubira, F. F., Mulira, N. (2004). Integration of ICT in organisations: Challenges and best practice recommendations based on the experience of Makerere University and other organizations. In: Present. to Conf. "Universities Tak. a Lead. role ICT enabled Hum. Dev. 
Vignovic, J. A., Thompson, L. F. (2010). Computer-mediated cross-cultural collaboration: Attributing communication errors to the person versus the situation. In: J. Appl. Psychol., 95(2), p. 265.

Workman, M., Kahnweiler, W., Bommer, W. (2003). The effects of cognitive style and media richness on commitment to telework and virtual teams, J. Vocat. Behav., 63(2), 199-219.

Yan Hong Lim, K., Zheng, P., Chen, C.-H. (2020). A state-of-the-art survey of Digital Twin: techniques, engineering product lifecycle management and business innovation perspectives. In: Journal of Intelligent Manufacturing, August.

Received February 18, 2021, revised May 5, 2021, accepted May 31, 2021 